\title{
Análise do conforto ambiental e ergonômico em uma instituição de ensino
}

\author{
Eduardo Nunes de Magalhães \\ Engenheiro Agrícola e Engenheiro de Segurança do Trabalho. \\ Professor do Ensino Básico Técnico e Tecnológico. Instituto Federal do Triângulo Mineiro Campus Curso \\ Técnico em Mineração. Patos de Minas, MG \\ $\checkmark$ eduardonunes@iftm.edu.br \\ Ester Melo Vieira \\ Aluna do Ensino Básico Técnico e Tecnológico. \\ Curso Técnico em Mineração. Instituto Federal do Triângulo Mineiro Campus Patos de Minas, MG \\ $\triangle$ estermelovieira@yahoo.com.br
}

\section{Resumo:}

O senso comum para o trabalho tem sido de punição, desgaste ou de exploração, o que vem mudando nos últimos anos graças aos investimentos acadêmicos e empresariais na busca pela melhoria na relação do homem com sua atividade laboral. Essa reorganização do pensamento e das atitudes com relação ao trabalho é motivada pela busca de uma qualidade de vida associada ao conforto ambiental e ergonômico, que buscam como objetivo primordial diminuir ou eliminar o aparecimento de doenças ocupacionais advindos das atividades relacionadas ao desempenho de uma função trabalhista. Nesse sentido, o termo conforto ambiental e ergonômico denotam a interdisciplinaridade entre diversos fatores, tais como: condições do ambiente de trabalho, familiar, condições psicossomáticas prévias ou adquiridas ao longo da vida, a fim de se obter uma correlação que permita ao trabalhador conhecimento suficiente para produzir qualidade de vida, e uma satisfação natural no desenvolvimento de suas atividades. O presente trabalho aborda, em específico, a atividade dos profissionais de educação; tanto técnicos administrativos quanto docentes no ambiente do Instituto Federal do Triângulo Mineiro; Campus Patos de Minas; por meio da aplicação de uma técnica conhecida como Análise Ergonômica do Trabalho - AET . Essa técnica consiste na utilização de entrevistas e análises físicas do ambiente (IBUTG, ruídos e iluminância) através de medições padronizadas pelas Normas Regulamentadoras 17 e 15 do Ministério do Trabalho. Percebeu-se que, no geral, a condição dos servidores é satisfatória, porém, há também um desconhecimento referente à ergonomia pelos técnicos administrativos e professores. Demonstrouse que o mobiliário, no geral, apresenta-se fora dos padrões recomendado, e que quanto as variáveis físicas medidas apenas a temperatura encontra-se fora dos padrões recomendados pela norma. Para uma análise mais profunda faz se necessário que o estudo seja aplicado ao longo dos anos para melhor compreensão da atividade.

Palavras-chave: Ergonomia, Escola, Conforto Ambiental, Educação.

\section{Analysis of environmental and ergonomic comfort in a teaching institution}

\begin{abstract}
:
The common sense for work has been punishment, wear or exploitation, which has been changing in recent years thanks to academic and business investments in the search for improvement in the
\end{abstract}


relationship between men and their work activity. This reorganization of thinking and attitudes towards work is motivated by the search for a quality of life associated with environmental and ergonomic comfort that seek as a primary objective to diminish or eliminate the appearance of occupational diseases arising from activities related to the performance of a labor function. In this sense, the term environmental comfort and ergonomic denote the interdisciplinarity among several factors such as conditions of work environment, family, previous psychosomatic conditions or acquired throughout the life in order to obtain a correlation that allows the worker sufficient knowledge to produce quality of life and a natural satisfaction in the development of their activities. The present work specifically addresses the activity of educational professionals, both administrative technicians and teachers, in the environment of the Federal Institute of the Minas Gerais Triangle Mineiro Campus through the application of a technique known as Ergonomic Analysis of Work - AET. This technique consists of the use of interviews and physical analysis of the environment (IBUTG, noise and illuminance) through measurements standardized by Regulatory Norms 17 and 15 of the Ministry of Labor. It was noticed that in general the condition of the servers is satisfactory, however that there is also an ignorance regarding ergonomics by administrative technicians and teachers. It has been shown that furniture in general is out of the recommended standards and that as far as physical variables measured only the temperature is outside the standards recommended by the standard. For a deeper analysis it is necessary if the study is applied over the years for a better understanding of the activity.

Keywords: Ergonomics, School, Environmental Comfort, Education.

\section{Análisis del confort ambiental y ergonómico en una institución de enseñanza}

\section{Resumen:}

El sentido común para el trabajo ha sido de castigo, de desgaste o de explotación, lo que viene cambiando en los últimos años, gracias a las inversiones académicas y empresariales en la búsqueda de la mejora en la relación del hombre con su actividad laboral. Esta reorganización del pensamiento y de las actitudes con relación al trabajo está motivada por la búsqueda de una calidad de vida asociada al confort ambiental y ergonómico, que buscan como objetivo primordial disminuir o eliminar la aparición de enfermedades ocupacionales derivadas de las actividades relacionadas al desempeño de una función laboral. En este sentido, el término confort ambiental y ergonómico denota la interdisciplinaridad entre diversos factores, tales como: condiciones del ambiente de trabajo, familiar, condiciones psicosomáticas, previas o adquiridas a lo largo de la vida a fin de obtener una correlación que permita al trabajador conocimiento suficiente para producir calidad de vida, y una satisfacción natural en el desarrollo de sus actividades. El presente trabajo aborda, en específico, la actividad de los profesionales de educación tanto técnicos administrativos como docentes en el ambiente del Instituto Federal do Triângulo Mineiro, Campus Patos de Minas, por medio de la aplicación de una técnica conocida como Análisis Ergonómico del Trabajo - AET. Esta técnica consiste en la utilización de entrevistas y análisis físicos del ambiente (IBUTG, ruidos e iluminancia) a través de mediciones estandarizadas por las Normas Reguladoras 17 y 15 del Ministerio de Trabajo. Se percibió que, en general, la condición de los servidores es satisfactoria, pero, hay también un desconocimiento referente a la ergonomía por los técnicos administrativos y profesores. Se demostró que el mobiliario, en general, se presenta fuera de los estándares recomendados, y que en cuanto a las variables físicas medidas sólo la temperatura se encuentra fuera de los estándares recomendados por la norma. Para un análisis más profundo se hace necesario que el estudio se aplique a lo largo de los años para una mejor comprensión de la actividad.

Palabras clave: Ergonomía, Escuela, Confort Ambiental, Educación. 


\section{INTRODUÇÃo}

A palavra "trabalho" é tão antiga quanto a própria história humana, uma vez que seus primeiros relatos aparecem nos textos bíblicos e é definida como forma de punição ao homem devido ao pecado (GÊNESIS, 3-19). Ao longo de sua história a concepção de trabalho está associada a fardo, uma vez que o conceito atribuído é de que existe uma troca do seu esforço físico e mental por uma recompensa econômica conhecida como salário (MASSON et al., 2015). No entanto, vários pensadores já criaram uma série de teorias para descrever o comportamento humano frente aos seus anseios, dentre as quais podemos destacar a teoria da equidade (Homans, Adams), a teoria das necessidades (Maslow, McClelland, Alderfer), a teoria dos dois fatores (Herzberg, Mausner, Snyderman), a teoria do reforço (Skinner, Connellan), a teoria das expectativas ou instrumentalidade (Vroom), a teoria dos objetivos (Locke, Bryan), para mencionar apenas as mais influentes.

Dentre todas as teorias apresentadas, a teoria das necessidades de Maslow ainda hoje é uma das teorias sobre motivação mais conhecidas, embora seja atualmente questionada em termos de sua validade, pelo fato de estar apoiada em evidências muito restritas de pesquisas realizadas sem experimentação (BERGAMINI, 1997; ROBBINS, 2002). Sua grande contribuição deve-se, principalmente, à lógica intuitiva da teoria e à sua facilidade de compreensão (ROBBINS, 2002). Porém, há uma discussão entre Robbins que a considera como lógica intuitiva e Sampaio (2009) que trata como equivocada a interpretação da teoria de Maslow, por simplificar em demasia os conceitos de sua teoria das necessidades. Na verdade, a lógica da "hierarquia das necessidades", buscava demonstrar que o bem estar humano físico e psíquico depende do atendimento em uma ordem lógica das principais necessidades humanas, sendo divididas em fisiológicas, de segurança, sociais, de estima e de auto- realização que mostrava que o ser humano dependia de algo mais que simplesmente do trabalho e que, além disso, não se buscava no trabalho apenas recompensa econômica.

De acordo com Sampaio (2009), em diversas conferências realizadas pelo próprio Maslow destacava que as pessoas possuem diferentes necessidades de motivação. Na verdade, a complexidade do tema motivação é descrita tanto por Vries (1993) que diz que apesar de ser um dos temas mais abordados, é sem dúvidas um dos temas menos compreendido do comportamento organizacional, quanto por Herzberg (1997) para quem 
por meio da utilização da psicologia da motivação é muita complexa e o que se desenvolveu com algum grau de certeza é ainda muito pouco.

Herzberg (1968), mostrava que além do fator econômico existiam dois fatores: os higiênicos, que estavam relacionados com o ambiente de trabalho que tinham a função de reduzir ou impedir a insatisfação, e os fatores motivacionais, que estavam ligados a satisfação no trabalho, de outros subfatores como a realização, o reconhecimento, do próprio trabalho, da responsabilidade, da possibilidade de crescimento e do desenvolvimento ou progresso atestada anos mais tarde por Abergo (2014).

Na metade do século XX o termo ergonomia surge na França como um estudo prático para solução de problemas reais dentro da indústria com a proposta de melhorar a situação já instalada nas fábricas. Essa análise in loco da vida real do trabalho nas indústrias foi proposta por Suzanne Pacaud (1949) e Obrendame e Faverge (1955) ao mostrarem a dificuldade no cálculo inicial e final quando da criação de um posto de trabalho. Para a linha francesa, a ergonomia trata de abordar a tarefa e a atividade e, portanto, para o ergonomista francês toda a preocupação está na organização do trabalho, ou seja, o que faz, quem faz, e de que maneira poderia fazê-lo melhor (MONTMOLLIN, 1990; VIDAL, 2000; WISNER, 2004; SANTOS, 2006)

Como pode ser visto, a discussão sobre a qualidade de vida no trabalho é relativamente antiga, porém, os estudos que envolvem o conforto ambiental começam a ser delineados através do estudo sobre ergonomia com Almeida et al. (2014) que define como "o conjunto dos conhecimentos científicos relativos ao homem e necessários à concepção de instrumentos, máquinas e dispositivos que possam ser utilizados com o máximo de conforto, segurança e eficiência".

Nesse universo do trabalho, as profissões de técnicos administrativos e docentes são pouco valorizadas no Brasil, sendo isso senso comum conhecido por qualquer cidadão. Todavia, em pouquíssimos momentos da história brasileira foi administrada de forma séria e com responsabilidade, já que há tempos elas vêm sofrendo pressões e uma desvalorização crescente diante das dramáticas mudanças principalmente políticas, em que gestores dispostos ao máximo lucro se utilizando da máquina pública vêm perdendo o foco da importância nos avanços do setor educacional formado por esses profissionais. 
Segundo a Organização Internacional do Trabalho -OIT (1984) professores são todas as pessoas que, nos diversos estabelecimentos de ensino, estão encarregadas da educação dos alunos. Segundo Cristina (2016) o termo "professor" também se aplica aos diretores de escola, aos supervisores e todos aqueles que dão assistência ao professor em seu trabalho, por meio de orientação ou de ajuda direta, como é o caso dos técnicos administrativos. Todavia, os amplos estudos epidemiológicos nessa área, em nosso país, são escassos ou poucos conclusivos no que diz respeito à qualidade de vida no trabalho realizado por técnicos administrativos e docentes no ambiente escolar. Portanto, este estudo objetivou realizar uma análise ergonômica do trabalho dos técnicos administrativos e docentes do Instituto Federal do Triângulo Mineiro, no Campus Patos de Minas.

\section{METODOLOGIA}

O presente trabalho foi realizado no Instituto Federal do Triângulo Mineiro, Campus Patos de Minas, localizado na zona rural de Patos de Minas-MG. Para realização da Análise Ergonômica do Trabalho (AET) são utilizadas duas ferramentas. A primeira utilizada foi a confecção de uma planilha eletrônica (conforme anexo), com diversas perguntas sobre as condições de trabalho, ambiente de trabalho e características pessoais para diagnóstico da percepção pessoal de cada servidor pelo tema proposto (Conforto Ambiental). A segunda ferramenta utilizada foi medições em cada local de trabalho de cada servidor do campus, focado na análise do mobiliário do ambiente de trabalho e em medições físicas (IBUTG, iluminância e ruído), de acordo com os padrões estabelecidos pela Norma Regulamentadora do Ministério do Trabalho ํㅜㄴ 17 intitulada Ergonomia.

\section{Condições climáticas}

A avaliação da exposição a temperaturas excessivas é bastante importante para que se possa garantir o conforto térmico do funcionário. Existem vários índices para avaliação da exposição ao calor, dentre os quais são destacados pelas normas regulamentadoras os 
Índice de Temperatura Efetiva Corrigida, Índice de Sobrecarga Térmica, Índice do Termômetro de Globo Úmido, Índice de Bulbo Úmido e o Termômetro de Globo (IBUTG).

\section{Índice de bulbo úmido termômetro de globo (IBUTG)}

Para a amostragem e posterior determinação do IBUTG foi utilizado um termômetro digital de Índice de Bulbo Úmido Termômetro de Globo (IBUTG), da marca Metrosonics, modelo HS - 3600. Esse índice representa o efeito combinado da radiação térmica, da temperatura de bulbo seco, da umidade e da velocidade do vento.

As avaliações de calor efetuadas através do IBUTG estão sujeitas a variações climáticas que dependem das estações do ano. No entanto, para efeito da estipulação de medidas preventivas, devem ser consideradas sempre as piores condições ambientais (COX, 1973, p. 692). De acordo com a NR-15, as equações que definem o IBUTG são as que seguem. Para ambientes internos ou externos sem carga solar (sem radiação solar direta):

$$
\mathrm{IBUTG}=0,7 \mathrm{tbn}+0,3 \mathrm{tg}
$$

Onde:

$$
\begin{gathered}
\text { tbn = temperatura de bulbo úmido natural } \\
\text { tg = temperatura de globo }
\end{gathered}
$$

Para a maior precisão possível foram realizadas leituras de hora em hora durante a jornada de trabalho na escola. A primeira leitura foi feita às $8 \mathrm{~h}$ e a última às $18 \mathrm{~h}$; elas aconteceram na sala de professores, na sala de aula, na sala dos técnicos administrativos e nos corredores.

\section{Níveis de ruído}

O ruído foi medido com o uso de um decibelímetro digital de marca Instrutherm, modelo DEC - 460, com sensor posicionado no meio da sala de aula, sala dos técnicos, sala de professores e corredor conforme a Norma Regulamentadora NR 15 (2006). Visando estabelecer o número mínimo de amostras necessárias, os dados foram coletados em 
intervalos de 20 em 20 segundos durante toda a realização da atividade específica, sendo encontrados os valores em decibéis $\mathrm{dB}(\mathrm{A})$ que foram lidos e anotados para, posteriormente, ser feita uma média do nível de ruído no ambiente de trabalho.

\section{Iluminância}

A iluminância foi medida com um luxímetro digital portátil de marca TES, modelo TES1332A. As leituras foram realizadas a cada 15 min, segundo a NBR 5413/92, na altura das mesas de trabalho e das carteiras dos alunos.

Os resultados obtidos dos níveis de ruído e iluminância foram submetidos a testes estatísticos por meio de uma análise de variância em que as médias obtidas pelos instrumentos de medição foram comparadas pelo teste de Tukey a 5\% de probabilidade, quando essas apresentaram diferenças estatísticas significativas.

\section{RESULTADOS E DISCUSSÕES}

Participaram da pesquisa 15 pessoas do sexo feminino e 13 do sexo masculino, sendo que $64,3 \%$ possuem menos de 35 anos de idade e 53,6\% estão na faixa etária de 24 a 35 anos (tabela 1).

Tabela 1- Distribuição amostral por faixa etária

\begin{tabular}{cc}
\hline FAIXA ETÁRIA & \% DA POPULAÇÃo \\
\hline 18 a 23 & 10,7 \\
24 a 35 & 53,6 \\
36 ou mais & 35,7 \\
Total & 100 \\
\hline Fonte: Própria &
\end{tabular}


A pesquisa constatou que $67,9 \%$ dos entrevistados disseram que o mobiliário está adequado às atividades e 60,7\% acreditam que as cadeiras são adequadas, porém, 92,9\% disseram que suas cadeiras não possuem sequer encostos de braço. A demonstração do desconhecimento do reconhecimento ergonômico é revelada quando 53,6\% da amostra têm o olhar fora da linha da visão no monitor, 85,7\% não usam apoio de punho e 96,4\% não usam apoio de pés. Talvez, por 64,3\% da amostra ter menos de 36 anos entendamos por que a maioria dos entrevistados não perceba nenhuma doença provocada pela atividade laboral, uma vez que essas enfermidades aparecem em idades mais avançadas após vários anos de trabalho.

Muitas vezes os profissionais não percebem o padrão de adoecimento, uma vez que a maioria das doenças ocupacionais é caracterizada por terem uma evolução lenta e progressiva advinda de condições que perduram e promovem desgastes de forma gradativa diretamente relacionada às condições de trabalho (ESMAEILZADEH e CAPAN, 2014).

No entanto, apesar de não haver relato de doenças confirmadas e, portanto, efetivamente diagnosticadas tanto técnicos administrativos quanto docentes apresentam, em diferentes graus, dores de coluna, musculares, lombares e estresse. Grande parte das doenças relacionadas às patologias com dores de coluna, musculares e lombares estão associadas a má postura geradas seja pelo uso do mobiliário inadequado ou pela falta de cuidados do próprio funcionário (OLIVEIRA et al., 2015; PAULA et al., 2015).

E nestes problemas, alguns fatores físicos e/ou biomecânicos relacionados aos mesmos, como a manutenção de posturas estáticas e inadequadas (principalmente da coluna e dos membros superiores) além, é claro, do estresse, podem favorecer o surgimento dos distúrbios osteomusculares advindos do trabalho (DORTs) e, dessa maneira, prejudicar a saúde geral dos profissionais (ESMAEILZADEH e CAPAN, 2014).

Já o estresse é uma doença que está diretamente ligada a outras mais graves tais como depressão, ansiedade, nervosismo e crises de pânico e tem sido relatado em diversas pesquisas no meio escolar como Bogaert et al., (2014) que associou diferentes tipos de problemas mentais e físicos, bem como sua correlação com o trabalho; percebendo o desenvolvimento de doenças sérias nestes profissionais com o passar do tempo.

Nesse quesito, as doenças psicossomáticas e as doenças de má postura estão também associadas diretamente à satisfação que temos no trabalho, uma vez que há uma melhora 
nas condições psicossomáticas do ser humano a medida que haja uma interação positiva do trabalho ao ser humano (LIMA et al., 2014). Segundo a pesquisa, a associação entre o volume excessivo de trabalho, juntamente com o ritmo acelerado de trabalho correspondem isoladamente por 53,6\% das razões para o aumento do nível de estresse no ambiente do IFTM Campus Patos de Minas.

Em compensação ao ritmo acelerado e o excesso de trabalho, os resultados evidenciam que 53,6\% gostam do trabalho que faz devido a oportunidade de aprender novas tarefas, o que demonstra que esses profissionais, na verdade, são amantes do trabalho; o que corrobora com Dias et al., 2013, mas, que a gestão dos recursos principalmente humanos é feita de forma errônea pelos gestores (ROSAS e MORAES, 2011; WITT et al., 2013).

Apesar de amarem o trabalho, no caso dos técnicos administrativos e dos docentes, um fator tem sido considerado bastante escasso e muito mal dimensionado pelos gestores que é o tempo. Na análise dos docentes, ainda tem sido pior já que não se tem calculado bem o tempo, principalmente no que tange as funções extraclasse, uma vez que os professores afirmaram levar trabalhos escolares para serem concluídos em casa, principalmente nos finais de semana. Como o tempo fica escasso, surge a necessidade de uma escolha por parte dos professores no que tange a priorização do tempo, o que na maioria das vezes é feito através do abandono da própria família e amigos, levando ao abandono de atividades físicas e práticas esportivas, comprometendo significativamente a qualidade de vida e, consequentemente, de uma prestação de excelência a educação (NARDI e SCHENEIDER, 2014).

Outra razão de grande estresse no que diz respeito aos docentes é a inversão de valores que tem ocorrido, pois a sociedade tem terceirizado para a escola a educação dos filhos. Atitude que gera desinteresse dos alunos pelo ensino, levando, muitas vezes, a uma exigência extrema de conhecimento e perssuasão para mostrar a importância e a necessidade de estudar. Essa situação leva educadores a níveis insuportáveis de problemas psíquicos e físicos além de gerar uma frustração nos estudantes uma vez que não conseguem assimilar como deferiam os conhecimentos e todo o aprendizado, levando muitos discentes a problemas até mesmo de autoestima (BATISTA et al., 2013). 
Essa ausência da presença familiar compromete o trabalho no ambiente escolar, pois há uma compreensão entre os professores de que os pais ou responsáveis deveriam participar de forma efetiva na vida escolar de seus filhos. Como consequência direta dessa ação, existe a multiplicação do trabalho realizado pelos profissionais (CRISTINA, 2016). Desse modo, percebe-se que os processos de saúde-doença dos profissionais da educação têm um conjunto de determinações sociais, políticas, culturais e individuais correlacionadas às condições de trabalho determinadas pela ausência familiar, uso de drogas, falta de condições de trabalho, governo que não privilegia a educação, etc.. Além dos fatores humanos apresentados, há outros fatores a serem considerados no estudo da ergonomia que estão relacionados às condições ambientais tais como a temperatura, umidade, ruídos, etc.

\section{Condições climáticas}

As salas de aula e a sala dos professores apresentaram temperatura de pico e temperaturas médias superiores ao corredor e a sala dos técnicos devido ao posicionamento do prédio onde o sol incide diretamente sobre uma parede constituída de vidro. Os resultados do IBUTG médio entre os horários de $8 \mathrm{~h}$ e término às $1 \mathrm{~h}$ tiveram uma média que segundo a NR 15 (SEGURANÇA e MEDICINA DO TRABALHO, 2006), é perfeitamente tolerada pelo organismo humano para esse tipo de atividade, considerada leve, permitindo um trabalho contínuo para uma jornada de $8 \mathrm{~h}$ de trabalho diárias (figura 1).

A Norma Regulamentadora NR 17, no seu item 17.5.1, dispõe que as condições ambientais de trabalho devem estar adequadas às características psicofisiológicas dos trabalhadores e à natureza do trabalho a ser executado. No item 17.5.2 da supracitada norma, nos locais de trabalho onde são executadas atividades que exijam solicitação intelectual e atenção constantes, tais como: salas de controle, laboratórios, escritórios, salas de desenvolvimento ou análise de projetos, dentre outros, é recomendado o índice de temperatura efetiva entre $20^{\circ} \mathrm{C}$ (vinte) e $23^{\circ} \mathrm{C}$ (vinte e três graus centígrados). Desta forma, as temperaturas dentro das salas de aula e na sala dos professores estão fora dos padrões adequados e poderiam ser solucionados com instalação de ares condicionados. 
Figura 1 - Temperaturas IBUTG para diferentes ambientes
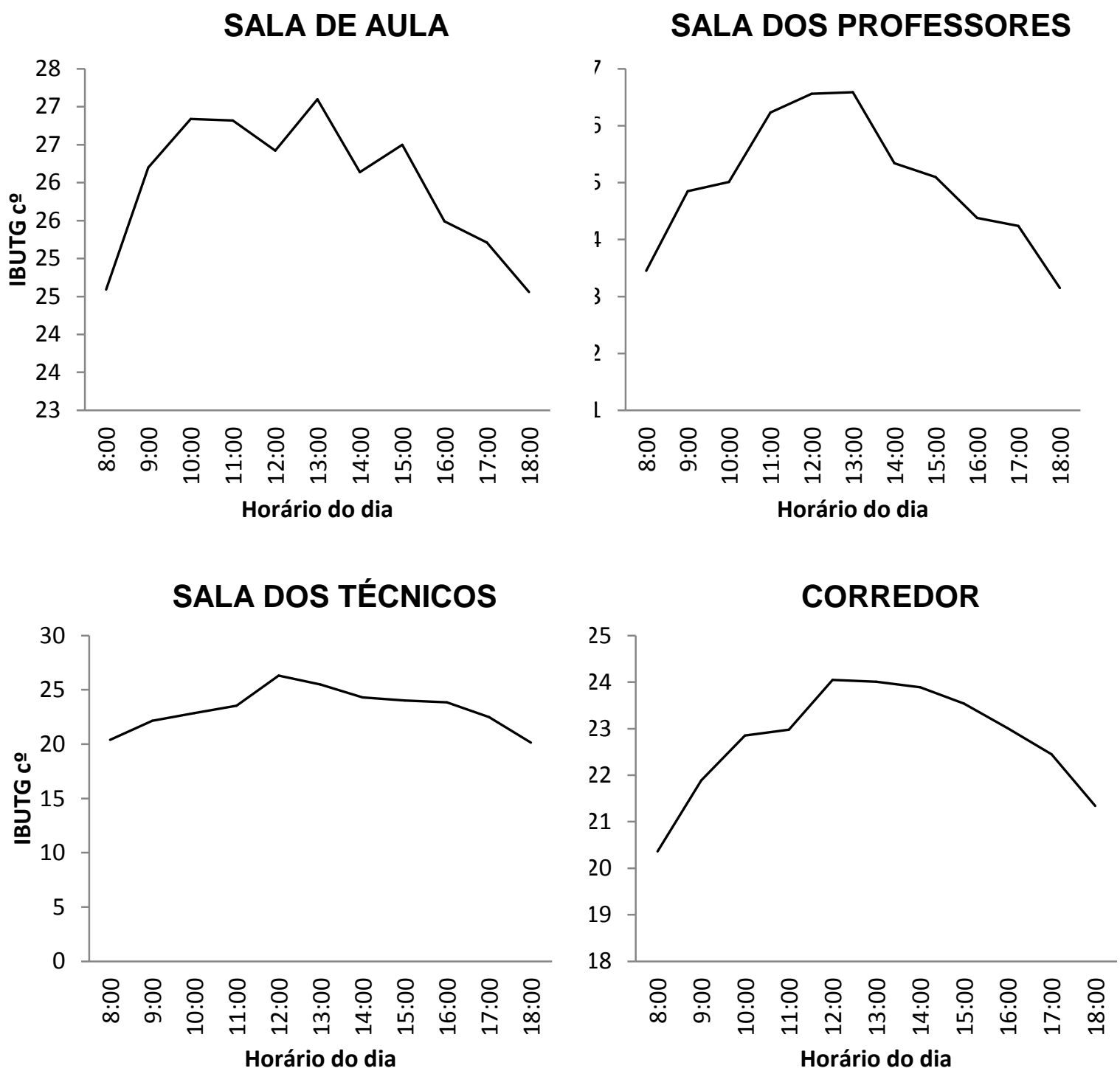

Fonte: Própria

\section{Níveis de ruído}

De acordo com a NR 15, para 8h de trabalhos diários, sem a utilização de protetor auricular, é permitido um máximo de exposição de $85 \mathrm{~dB}(\mathrm{~A})$. Os resultados demonstram que os nível máximo atingido no corredor esta fora do padrão recomendado em valor nominal, porém, são de curta duração e, normalmente, sem presença de funcionários diretamente expostos. Todos os outros ambientes escolares não possuem valores de ruído fora dos padrões recomendados (tabela 2). 
Tabela 2- Nível de ruído em diferentes ambientes da escola.

\begin{tabular}{cccc}
\hline & \multicolumn{2}{c}{ NÍVEL DE RUÍDO } & \\
\cline { 2 - 3 } LOCAL & MÍNIMO & MÁXIMO & $\begin{array}{c}\text { EXPOSIÇÃo } \\
\text { DIÁRIA } \\
\text { PERMITIDA } \\
\text { (HORAS) }\end{array}$ \\
\hline Sala de aula & 66,10 & 85,20 & 4 \\
Sala de professores & 61,40 & 78,00 & 8 \\
Corredor & 78,45 & 95,20 & 1 \\
$\begin{array}{c}\text { Sala de Técnicos } \\
\text { administrativos }\end{array}$ & 45,25 & 75,24 & 8 \\
\hline
\end{tabular}

Fonte: Própria

\section{Níveis de iluminância}

De acordo com a NBR 5413/92 (iluminação geral para áreas de trabalho onde exige tarefa com requisitos visuais limitados), salas de aulas, sala de professores e sala dos técnicos administrativos, a iluminância mínima recomendada deve ser de 200 a 500 lux. A iluminância média encontrada nos ambientes da escola está disposta conforme na tabela 3.

Tabela 3- Taxas de iluminamento para diferentes ambientes.

\begin{tabular}{cc}
\hline LOCAL & MÉDIA LUX \\
\hline Sala de aula & 650 \\
Sala de professores & 625 \\
Corredor & 560 \\
Sala de Técnicos administrativos & 600 \\
Fonte: Própria &
\end{tabular}

Portanto, de acordo com a norma, os ambientes contemplam uma iluminância superior à estabelecida pela norma. Existem dois fatores importantes na iluminação que são a luz suficiente no posto de trabalho e a eliminação completa de qualquer brilho que 
provoque ofuscamento, que é facilmente resolvida com pinturas foscas como as da escola em estudo e, portanto, dentro dos padrões.

\section{Índice de massa corporal}

Diversos estudos têm demonstrado a ligação entre a ansiedade, a depressão e outras doenças psíquicas como a compulsão alimentar; seja pela diminuição ou pelo aumento exacerbado de consumo de alimentos. Um dos ambientes mais propícios a essa promoção é o local de trabalho, onde tem-se uma permanência considerável e, nesse sentido, o ambiente escolar pode ser um gerador de mal estar e, portanto, o desencadeador de distúrbios alimentares.

Nessa perspectiva, é imprescindível o estudo dos índices que possuam ligação entre causa e efeito no estudo da ergonomia como é o caso do Índice de Massa Corporal - IMC peso em quilogramas dividido pelo quadrado da altura em metros - como instrumento de estimativa da obesidade. Esse índice tipifica numericamente as classificações obtidas em função do grau de magreza ou de obesidade. Este é o índice usado na classificação da OMS para o sobrepeso, conforme a tabela 4.

Tabela 4 - Valor do IMC e da Classificação dos Graus de Obesidade

\begin{tabular}{ccl}
\hline IMC & VALOR & CLASSIFICAÇÃo \\
\hline$<16$ & 0 & Magreza grau III \\
$16-16,99$ & 0 & Magreza grau II \\
$17-18,49$ & 0 & Magreza Grau I \\
$18,5-24,99$ & 17 & Normal \\
$25-29,99$ & 10 & Sobrepeso \\
$30-34,99$ & 1 & Obesidade grau I \\
$35-39,99$ & 0 & Obesidade grau II \\
$>40$ & 0 & Obesidade grau III \\
\hline
\end{tabular}

Fonte: Organização Mundial da Saúde (1995,1997). 
De acordo com a tabela e a classificação utilizada, cerca de $40 \%$ dos servidores possuem índices de massa corporal superiores ao recomendado pela organização mundial da saúde. A obesidade pode ser desencadeada por diversos fatores, tais como por doenças genéticas ou endócrinas quanto por influência de fatores ambientais, como mudança de hábitos alimentares e baixa atividade física (JOHNSTON et al., 2014) o que corrobora com os dados apresentados na tabela 5, que demonstra que cerca de $42 \%$ dos entrevistados não praticam nenhuma atividade física.

Tabela 5 - Frequência da atividade física na amostra estudada

\begin{tabular}{cc}
\hline FREQUÊNCIA & VALORES \\
\cline { 2 - 2 } Não pratica nenhuma atividade física & 12 \\
Pratica 1 vez por semana & 1 \\
Pratica 2ou 3 vezes por semana & 9 \\
Pratica de 4 a 5 vezes por semana & 6 \\
\hline
\end{tabular}

Fonte: Própria

A obesidade exógena ou por ingestão calórica reflete o excesso de gordura decorrente do balanço positivo de energia entre a ingestão e o gasto calórico, sendo responsável por 95\% dos casos de obesidade" (BARBIERE et al., 2012). A obesidade é apresentada como importante fator para o desenvolvimento de doenças crônicas e que podem ser desencadeadas, por exemplo, pelas condições de trabalho. Há uma tendência de considerar doenças cardiovasculares, relacionadas com a obesidade (CARVALHO et al., 2016), como patologias relacionadas ao trabalho, uma vez que apesar de ser fator preponderante, não deve ser negligenciado, mas também não se tornar explicação para todos os casos. No entanto, como existe a relação de nexo causal ligado ao trabalho, as empresas devem aumentar a preocupação com seus servidores, obrigando-os a adotarem medidas mais amplas de prevenção (CAMPOS, 1995). Com o advento da obesidade, há um aumento da prevalência em diferentes populações do mundo, que comprovadamente pode levar a problemas mais sérios, com elevado grau de mortalidade por estar associada a doenças como hipertensão arterial e diabetes, depressão, estresse, compulsão alimentar, dentre outras. 


\section{CONSIDERAÇÕES FINAIS:}

O conforto ambiental e ergonômico ainda é tratado de forma equivocada, já que não apresenta o imediatismo nas respostas, ou seja, todo trabalhador independentemente da atividade laboral desenvolvida possuem algum conhecimento sobre os temas e sua importância, mas desconhece os fatores promotores e o fato de que acontecem de forma silenciosa e lenta, surgindo, muitas vezes, no final das carreiras profissionais.

Como sugestão pertinente é indicado que esse estudo seja repeito posteriormente a fim de verificar se houveram mudanças significativas nos acontecimentos, principalmente aos ligados às doenças profissionais que tangem os servidores da educação, para que não surjam doenças ligadas a esse ambiente escolar, e isso será buscado por meio da divulgação dos resultados e conscientização da comunidade escolar.

\section{REFERÊNCIAS}

ABERGO- Associação Brasileira de Ergonomia. o que é Ergonomia. 2014. Disponível em: http://www.abergo.org.br/internas.php?pg=o_que_e_ergonomia.

ALMEIDA M.A.B.; GUTIERREZ, G.L.; MARQUES, R.. Qualidade de vida: definição, conceitos e interfaces com outras áreas de pesquisa. São Paulo: Escola de Artes, Ciências e Humanidades-EACH/USP; 2014.

BARBIERE.A.F.; NOBRE, M. R. C.; DOMINGUES, R. Z. L.; SILVA, A.. As causas da obesidade: Uma análise sobre a perspectiva materialista histórica. : revista da Faculdade de Educação Física da UNICAMP, Campinas, v. 10, n. 1, p. 133-153, jan./abr. 2012. ISSN: 1983-9030 2012.

BATISTA, J.B.V.; CARLOTTO, M.S.; MOREIRA, A.M.. Depressão como causa de afastamento do trabalho: um estudo com professores do ensino fundamental. Revista de Psicologia. 2013.

BERGAMINI, Cecília Whitaker; CODA, Roberto. (Org.). Psicodinâmica da vida organizacional: motivação e liderança. 2. ed. São Paulo: Atlas, . p. 69-93, 1997.

BRAVERMAN, H.. Trabalho e capital monopolista: a degradação do trabalho no século XX. Rio de Janeiro, Zahar, 1974.

BOGAERT I, M. K.; DEFORCHE, B.; CLARYS, P.; ZINZEN E.. Associations between different types of physical activity and teachers' perceived mental, physical, and work-related health. 2014. Disponível em: https://bmcpublichealth.biomedcentral.com/articles/10.1186/1471-2458-14-534 . Acesso em 01 de dezembro de 2017.

CAMPOS, A. L. R.. Aspectos psicológicos da obesidade.. São Paulo: Fundo Editorial BYK. 1995.

CARVALHO, F. J. P.; CIPULLO, M.A.T.; CARANTI, M..C.L.; ANDRADE,S.S.G.; PISANI. L.P.. Interdisciplinary lifestyle therapy improves binge eating symptoms and body image dissatisfaction in Brazilian obese adults. 2016. Disponível em: http://www.scielo.br/scielo.php?script=sci_arttext\&pid=S223760892012000400008. Acesso em 01 de novembro de 2017. 
CRISTINA, V. P. R. A relação trabalho-família: as condições de vida e de trabalho influenciam a qualidade do ambiente familiar?. $2016 . \quad$ Disponível educativo https://comum.rcaap.pt/bitstream/10400.26/17793/1/Cristina\%20Vanessa\%20Paiva\%20Ramos.pdf. Acesso em 01 de novembro de 2017.

COX, Joe W. Temperaturas Extremas. In: FUNDACENTRO. Curso para Engenheiros de Segurança do Trabalho. 1973. Disponível em: http://www.fundacentro.gov.br/biblioteca/detalhe-acervofisico?p=aHR0cDovL3NlcnZlcmJsL25ldGFjZ2kvcmVuaS5leGU/U0VDVDE9SU1BR0UmU0VDVDQ9ZSZTRUNUNj1I SVRPRkYmU0VDVDU9QklCTDAyJmQ9QklCTCZwPTkyNCZ1PWFjZXJ2by1maXNpY28mcj0xODQ3MiZmPUcmczE 9JnMyPW1vbm9ncmFmaWEudGlwby4rbmFvK2NvbmZpZGVuY2lhbC50aXBvLitvdSthbmFsaXRpY2EudGlwby4r bmFvK2NvbmZpZGVuY2lhbC50aXBvLitvdSttYXRlcmlhbC50aXBvLitvdSthcnRpZ28udGlwby4rb3UrY29sZWNhb y50aXBvLituYW8rY29uZmlkZW5jaWFsLnRpcG8uJmw9MjAmU0VDVDI9VEhFU090JlNFQ1QzPVBMVVJPTg== ... Acesso em 01 de dezembro de 2017.

DIAS, D.D.S.; BARROS, M.E.D.; SILVA, O.I.V.; CAMPOS, G.M.. Das dores e delícias do trabalho docente: uma análise do trabalho educação. $2013 . \quad$ Disponível https://seer.ufmg.br/index.php/trabedu/article/view/7332. Acesso em 01 de novembro de 2017.

DUNCAN, B.B.; CHOR, D.; AQUINO, E.M.; BENSENOR, I.M.; MILL, J.G.; SCHMIDT, M.I.. Chronic noncommunicable diseases in Brazil: priorities for disease management and research. 2012. Disponível em: http://www.scielo.br/scielo.php?script=sci_arttext\&pid=S0034-89102012000700017 . Acesso em 01 de dezembro de 2017.

ESMAEILZADEH S. O., CAPAN N.. Effects of ergonomic intervention on work-related upper extremity musculoskeletal disorders among computer workers: a randomized controlled trial. 2014. Disponível em: https://www.ncbi.nlm.nih.gov/pubmed/23263694. Acesso em 01 de dezembro de 2017.

FRIEDMANN, G. O futuro do trabalho humano. Editora não identificada.Lisboa. 1968.

HERZBERG, F. I. One more time: how do you motivate employees?. Harvard Business Review. Boston. v. 46, p. 53-62. $1968 . \quad$ Disponível em: https://www.researchgate.net/publication/10934893_One_More_Time_How_do_You_Motivate_Employees . Acesso em 01 de dezembro de 2017.

HERZBERG, F. Novamente: como se faz para motivar funcionários? . 1997. Disponível em https://books.google.com.br/books/about/Novamente.html?id=59lCtwAACAAJ\&redir_esc=y . Adquirido em 01 de setembro de 2017.

JOHNSTON V;O'LEARY, S.; COMANS, T.; STRAKER, L.; MELLOH, M.; KHAN, A.; SJOGAARD, G.. A workplace exercise versus health promotion intervention to prevent and reduce the economic and personal burden of non-specific neck pain in office personnel: protocol of a cluster-randomised controlled trial.2014. Disponível em: https://www.ncbi.nlm.nih.gov/pubmed/25306220 . Acesso em 01 de dezembro de 2017.

JUDGE; KAMMEYER, M.. Job Attitudes. Rev. psychol, v.1, p 341-367. 2012. Disponível em: https://www.ncbi.nlm.nih.gov/pubmed/22129457. Acesso em 01 de dezembro de 2017.

LIMA L ; PIRES, D.; FORTE, E.C.N.; MEDEIROS, F.. Job satisfaction and dissatisfaction of primary health care professionals. Esc Ana Nery Revista de Enfermagem, 2014; 18(1): 17-24, jan./mar.

MASSON, V.A.; MONTEIRO, M.I.; VEDOVATO, T.G.. Workers of CEASA: factors associated with fatigue and work ability. Rev Bras $2015 . \quad$ Disponível http://www.scielo.br/scielo.php?script=sci_arttext\&pid=S0034-71672015000300460 . Acesso em 01 de dezembro de 2017.

MASLOW, ABRAHAM H. Motivation and personality. New York, Harper \& Row, p 369, 1970. Disponível em: http://www.cra-rj.adm.br/publicacoes/textos_classicos/Motivation_and_Personality_Traduzido. Acesso em 01 de dezembro de 2017. 
MONTMOLLIN, M. A Ergonomia. Lisboa: Instituto Piaget, 1990. Disponível em: https://www.fnac.pt/AErgonomia-Maurice-de-Montmollin/a528036. Adquirido em 01/09/2017.

NARDI, E.L, SCHNEIDER, M.P.. Condições de trabalho docente: novas tessituras das políticas de avaliação para a qualidade. Educação, v.01, p.39.2014. Disponível em: https://periodicos.ufsm.br/reveducacao/article/view/9123. Acesso em 01 de dezembro de 2017.

NORMA REGULAMENTADORA NR-15 - Atividades e Operações Insalubres. 2015. Disponível em: http://portal.mte.gov.br/legislacao/normas-regulamentadoras-1.htm. Acesso em 01 de dezembro de 2017.

NORMA REGULAMENTADORA NR-17 - Ergonomia. Disponível em: http://portal.mte.gov.br/legislacao/normas-regulamentadoras-1.htm. Acesso em 01 de dezembro de 2017.

OLIVEIRA, M.M.; ANDRADE, S.S.C.A.; SOUZA, C.A.V.; PONTE, J.N.; SZWARCWALD, C.L.; MALTA, D.C.. Problema crônico de coluna e diagnóstico de distúrbios osteomusculares relacionados ao trabalho (DORT) autorreferidos no Brasil: Epidemiol Serv Saude, v.01, p 96-287, 2015. Disponível em:. https://doi.org/10.5123/S1679-49742015000200011. Acesso em 01 de dezembro de 2017.

PAULA, IR.; MARCACINE, P.R.; CASTRO, S.S.; WALSH, I.A.P.. Capacidade para o trabalho, sintomas osteomusculares e qualidade de vida entre agentes comunitários de saúde em Uberaba, Minas Gerais. Saúde Soc, v.1, p 64-152, 2015. Disponível em: http://www.scielo.br/scielo.php?pid=S0104$12902015000100152 \&$ script=sci_abstract . Acesso em 01 de dezembro de 2017

ROBBINS, S. Comportamento organizacional. São Paulo: Prentice Hall, 2002.

ROSAS, M.L.M.; MORAES, R.D.. A importância do reconhecimento no contexto de trabalho. Revista Amazônica. Humaitá, p 210-224, 2011. Disponível em: https://dialnet.unirioja.es/servlet/articulo?codigo=3915959. Acesso em 01 de dezembro de 2017.

SAMPAIO, J. R. O Maslow desconhecido: uma revisão de seus principais trabalhos sobre motivação. Revista de Administração da USP, v. 44, v.1, p 5-16, 2009.

SANTOS, R. L. G. Usabilidade de interfaces para sistemas de recuperação de informação na web: estudo de caso de bibliotecas on-line de universidades federais brasileira. 2006. Disponível em: https://www.maxwell.vrac.puc-rio.br/Busca_etds.php?strSecao=resultado\&nrSeq=9731@1. Acesso em 01 de dezembro de 2017.

TRIST, E. L. \& BAMFORTH, K. W. Some social and psychological consequences of the longwall method of coal-getting. 1951. Disponível em: http://journals.sagepub.com/doi/abs/10.1177/00187267510040010 . Acesso em 01 de dezembro de 2017.

VIDAL, M. C. Introdução à Ergonomia. 2000. Disponível em: <http:// www.ergonomia.ufpr.br/Introducao\%20a\%20Ergonomia\%20Vidal\%20CESERG. Acesso em 01 de dezembro de 2017.

VRIES, M.K. A inveja, a grande esquecida dos fatores de motivação em gestão. Atlas, 1993.

WITT, L.B.; OLSEN, D.; ABLAH, E. Motivating factors for small and midsized businesses to implement worksite health promotion. Saúde Promot Pract. 2013. Disponível em: https://www.ncbi.nlm.nih.gov/pubmed/23345340. Acesso em 01 de dezembro de 2017.

WISNER, A. A inteligência do trabalho. 1994. Disponível em https://www.estantevirtual.com.br/livros/alainwisner/a-inteligencia-no-trabalho/1587518105. Adquirido em 01 de setembro de 2017.

WOODWARD, J. Industrial organizations: theory and practice. Oxford University Press, 1965. Disponível em: https://books.google.com.br/books?hl=pt-

BR\&lr=\&id=xIuTDAAAQBAJ\&oi=fnd\&pg=PP1\&dq=Industrial+organizations:+theory+and+practice\&ots=RuVKPz As0y\&sig=AzhkG2NQ8nfiv29TQ3tbtT-

Vn1w\#v=onepage\&q=Industrial\%20organizations\%3A\%20theory\%20and\%20practice\&f=false. Acesso em 01 de dezembro de 2017. 


\section{ANEXO \\ QUESTIONÁRIO: ANÁLISE ERGONÔMICA DO TRABALHO-AET}

1. Como você considera o mobiliário de seu ambiente de trabalho? ótimo bom regular péssimo

2. As cadeiras são adequadas às atividades do seu dia-a-dia? Qual a nota você daria de 0 a 10 sabendo-se que 0 é péssimo e 10 excelente.

3. O encosto da cadeira fica na posição média das suas costas?

4. — $\operatorname{sim} \_$não

5. As cadeiras possuem braço? $\operatorname{sim}$ não

6. Quantas vezes no período de trabalho de 8 horas você costuma levantar e fazer uma pequena caminhada?

7. Como se dá a troca de informação no seu ambiente de trabalho? verbal

_ por intermédio de telefone

documentais

através de gestos

8. Quando você está diante do computador qual é a direção do olhar? acima da linha de visão

na linha da visão

abaixo da linha da visão

o limite superior da tela fica abaixo da linha de visão

o limite inferior da tela fica acima da linha de visão

9. Qual é a posição do seu punho durante a digitação? neutro (reto) com posição flexionada para cima com posição flexionada para baixo em balanço 
encurvado

10. Com qual frequência você costuma descansar a visão, olhando para outros objetos? nunca

01 a 02 vezes a cada 2 horas

03 a 04 vezes a cada 2 horas

05 a 06 vezes a cada 2 horas

07 a 08 vezes a cada 2 horas

11. Você usa apoio de punho?

nunca

1 dia por semana

2 dias por semana

3 dias por semana

4 dias por semana

todos os dias

12. Você usa apoio de pés?

nunca

1 dia por semana

2 dias por semana

3 dias por semana

4 dias por semana

todos os dias

13. Qual o nível de organização do seu ambiente de trabalho?

nenhum ruim razoável satisfatório

Extremamente organizado

14. Durante os trabalhos de digitação qual a posição dos seus pés em relação ao chão? uso descanso de pés

paralelo ao piso

flexionado

apoia apenas o calcanhar

apoia apenas os dedos

15. Como é a iluminação do seu ambiente de trabalho?

extremamente forte __ótima __ boa __ regular _ péssima

16. Qual é a posição do seu computador em relação a sala que você trabalha?

próximo da janela __ afastado da janela __ próximo a porta próximo a equipamentos___ próximo a saídas de emergências

16. Há instalação de ar condicionado no seu setor de trabalho? $\operatorname{sim}$ não

17. Quais os tipos de ruídos há no seu ambiente de trabalho? sirenes alunos impressoras antigas 
central de computadores

telefones

ruídos externos

18. Com quantas pessoas você divide o ambiente de trabalho?

19. Qual seu sexo?

masculino feminino

20. Qual sua idade?

18 a $23 \_24$ a $35 \_36$ ou mais

21. Altura —

22. Peso $\mathrm{Kg}$

23. Há quanto tempo realiza essa atividade?

24. Trabalha quantas horas por dia? __ dia

25. O que é bom no trabalho para você?

26. O que é ruim no trabalho para você?

27. Você sente dores? Onde? Com que frequência? Já procurou ajuda médica para o problema?

28. Você se sente estressado com o trabalho? Por quê?

29. Você pratica alguma atividade física? Com que Frequência?

30. Qual seu nível de preocupação com a ergonomia fora do ambiente de trabalho? nenhuma baixo médio normal extremo

31. Qual aspecto abaixo você acredita ser mais negativo para aumentar o nível de estresse? Ritmo acelerado de trabalho 
__ Volume excessivo de trabalho

__ Tempo insuficiente para realização das tarefas

__ Solicitações conflitantes

__ Posição inadequada e incômoda do corpo

__ Ritmo frenético de trabalho

__ Posições da cabeça e braços inadequadas e incômodas

__ Longos períodos de intensa concentração em uma mesma tarefa

32. Qual aspecto abaixo você acredita ser mais positivo no seu ambiente de trabalho

_ Necessidade de ser criativo

__ Necessidade de um alto nível de habilidade

__ Possibilidade de aprender novas coisas

_ Colegas competentes em fazer suas atividades

__ Pessoas amigáveis no ambiente de trabalho

Possibilidade de dar opinião sobre o seu próprio trabalho

33. Qual o seu nível de satisfação em relação à adequação do quadro utilizado por você, à sua altura?

indiferente __ muito satisfeito __ satisfeito _ _ pouco satisfeito __ insatisfeito 